\title{
The Heart Shape-Sign in Bilateral Medial Medullary Infarction; Complicated with Quadriparesis and Respiratory Failure
}

\section{Liaquat Ali', Numan Amir, Naveed Akhtar, Osama Elalamy, Mohammed Alhatou, Gholam Adeli and Ambreen lqrar}

Department of Neurology, Hamad General Hospital, HMC, Doha, Qatar

*Corresponding author: Liaquat Ali, Hamad General Hospital, Department of Neurology, Dept HGH, al Rayyan road, PO box 3050, Doha, Qatar, Tel: +97444392768

\begin{abstract}
Introduction: $20 \%$ of ischemic strokes involve posterior circulation. The most common causes of posterior circulation large artery ischemia are atherosclerosis, embolism, and dissection. The most common location of atherosclerotic occlusive disease within the posterior circulation is the proximal portion of the vertebral artery in the neck within the first inches. Basilar artery occlusive disease most often presents as ischemia in the pons. Occlusion of the rostral portion of the basilar artery can cause ischemia of the midbrain, thalami, temporal, and occipital lobe. The most frequent finding in patients with PCA territory infarction is a hemianopia. Bilateral medial medullary stroke is a very rare. The characteristic MRI finding of "heart shape sign" at DWI has been described.

Method: 50-year-old gentleman presented with acute vertigo, vomiting, bulbar symptoms, quadriparesis, respiratory failure and obtunded. Patient was intubated to protect airway. MRI head showed bilateral medial medullary acute infarct ("heart shape sign") and occlusion of left distal vertebral artery V4 segment. Patient CSF analysis and viral HSV-PCR was normal. He was shifted to rehabilitation center and discharged to home with modified rankin scale of $2 / 6$.
\end{abstract}

Discussion: Medial medullary infraction is $<1 \%$ of vertebrobasilar strokes and bilateral stroke is very rare. This case study may help in early recognition of this type of stroke presenting with triad of bulbar symptoms, quadriparesis and respiratory failure.

Conclusion: Bilateral medial medullary stroke is very rare stroke. GBS and Neuromuscular disorders are important differential of bilateral medial medullary stroke presenting triad of bulbar symptoms, quadriparesis and respiratory failure. Majority of patient with bilateral medial medullary stroke who survivors remained dependent.

\section{Keywords}

Extracranial vertebral artery (ECVA), Intracranial vertebral artery (ICVA), Posterior cerebral artery (PCA), Lower motor neuron (LMN), Medial research council (MRC)

\section{Background}

Stroke is the second most common cause of mortality and morbidity, the incidence of stroke due to ischemia is $68 \%$, while of hemorrhagic stroke is $32 \%$, reflecting a higher incidence of hemorrhagic stroke in low- and middle-income countries. $20 \%$ of ischemic strokes in the brain involve posterior circulation structures (vertebrobasilar circulation). The most common causes of posterior circulation large artery ischemia are atherosclerosis, embolism, and dissection [1].

Atherostenotic lesions of the innominate and subclavian arteries cause arm ischemia and TIAs but seldom cause strokes. In subclavian steal syndrome, obstruction of the proximal subclavian artery produces a low-pressure system within the ipsilateral vertebral artery and in blood vessels of the ipsilateral upper extremity. Most patients with subclavian artery disease are asymptomatic. Innominate artery occlusive disease presented with ischemia to ipsilateral carotid artery, ipsilateral arm, and distal portion of the posterior circulation and/or cerebellum. Takayasu's disease and giant cell arteritis can cause subclavian and innominate artery occlusive disease $[2,3]$.

Most occlusive lesions of the proximal vertebral

Citation: Ali L, Amir N, Akhtar N, Elalamy O, Alhatou M, et al. (2021) The Heart Shape-Sign in Bilateral Medial Medullary Infarction; Complicated with Quadriparesis and Respiratory Failure. Clin Med Rev Case Rep 8:359. doi.org/10.23937/2378-3656/1410359

Accepted: July 30, 2021: Published: July 31, 2021

Copyright: (c) 2021 Ali L, et al. This is an open-access article distributed under the terms of the Creative Commons Attribution License, which permits unrestricted use, distribution, and reproduction in any medium, provided the original author and source are credited. 
arteries are atherosclerotic [4]. The most common location of atherosclerotic occlusive disease within the posterior circulation is the proximal portion of the vertebral artery in the neck within the first inch (2 to 3 $\mathrm{cm}$ ) of the ECVAs. In patients with proximal extracranial vertebral artery (ECVA) stenosis, intraarterial (arteryto-artery) embolism to the intracranial posterior circulation is a much more frequent cause of ischemia than hemodynamic insufficiency (low flow). Patients presenting with ischemia in the distribution of the intracranial vertebral arteries (the medulla and posterior inferior cerebellum) or the distal basilar artery (superior cerebellum, occipital and temporal lobes in the territory of the posterior cerebral arteries, or the thalamus or midbrain) show a high frequency of recent ECVA occlusions. Dissection of the ECVA usually involves the distal portion as it winds around the upper cervical vertebrae or proximal portion between origin of the artery and its entry into the vertebral column at $\mathrm{C} 5$ or C6 [5]. Atherostenotic disease can involve any portion of the intracranial vertebral arteries (ICVA). Occlusive ICVA disease presents in a variety of different ways including asymptomatic, TIAs, lateral or medial or hemi-medullary infraction, Cerebellar infarction in PICA territory [6]. Embolization of the ICVA thrombus to the distal basilar artery and its branches causing TIAs and/ or strokes [7].

Basilar artery occlusive disease most often presents as ischemia in the pons. The major burden of ischemia is in the middle of the pons, mostly in the paramedian base, and often also in the paramedian tegmentum [8]. Most patients with symptomatic basilar artery occlusive disease and pontine ischemia have some transient or persistent degree of paresis and corticospinal tract abnormalities. Bulbar symptoms include facial weakness, dysphonia, dysarthria, dysphagia, and limited jaw movements. Oculomotor symptoms and signs are common [9]. Occlusion of the rostral portion of the basilar artery (the "top of the basilar") can cause ischemia of the midbrain, thalami, and temporal and occipital lobe hemisphere territories supplied by the posterior cerebral artery branches of the basilar artery. Infarction in this region is typically caused by embolism from a more proximal source such as the heart, the aorta, the vertebral arteries in the neck, or the intracranial vertebral arteries. Less commonly, the syndrome is caused by intrinsic occlusive disease of the rostral portion of the basilar artery. The major abnormalities associated with rostral brainstem infarction involve alertness, behavior, memory, and oculomotor and pupillary functions [10].

Most posterior cerebral artery (PCA) territory infarcts are due to embolism from the heart, aorta, or vertebral arteries. The most frequent finding in patients with PCA territory infarction is a hemianopia [11]. Lateral thalamic infarction is the major reason for somatosensory symptoms and signs. Additional neurologic syndromes found with infarction of the left PCA territory include alexia without agraphia, anomic aphasia, or transcortical sensory aphasia, and Gerstmann syndrome (acalculia, agraphia, finger agnosia, and right-left disorientation). Syndromes observed with right PCA territory infarction include prosopagnosia, which is difficulty in recognizing familiar faces, spatial disorientation, and visual neglect [12].

In medial medullary stroke, the most consistent finding is a contralateral hemiparesis [13]. In approximately one-half of patients, the face is also involved. Facial weakness is usually slight and transient and rarely persists. Sensory symptoms are related to ischemia of the medial lemniscus. Ipsilateral tongue paralysis is the least common but most topographically localizing sign of medial medullary infarction and is due to involvement of the hypoglossal nucleus. Less than $1 \%$ of all posterior circulation infarctions present as medial medullary syndrome [14]. It results from the occlusion of one of the paramedian perforators of the anterior spinal artery or vertebral arteries. Medial medullary syndrome was first described by Spiller in the $19^{\text {th }}$ century. Dejerine then proposed a clinical trial of ipsilateral hypoglossal paralysis, contralateral hemiparesis sparing the face, and contralateral loss of deep sensation [15]. Bilateral medial medullary stroke is a very rare type of stroke. The most common symptoms are weakness, dysarthria, hypoglossal palsy, flaccid, or spastic quadriplegia. The characteristic brain MRI finding of "heart appearance" at DWI has been described in multiple case reports [16]. Normal MRA of brain may suggest small vessel disease of one of the paramedian perforating (from vertebral or anterior spinal arteries). It is possible if one paramedian artery is supplying both pyramids in rare cases. GBS can mimic the presentation, particularly with diffuse areflexia and progressive weakness, and can excluded by MRI findings. A keypoint to differentiate both syndromes is the evolution of the clinical presentation, whereas GBS have and sub-acute evolution while bilateral medial medullary infraction has a more acute presentation. The outcomes are poor, with a severe mortality and morbidity, in a systematic review including 38 patients with bilateral medial medullary infraction proven by MRI, one fourth of patients died and two third of survivors remained dependent (mortality, 23.8\%; dependency, 61.9\%) [17].

\section{Methodology}

\section{Case report}

50-year-old gentleman presented to ED with sudden onset of spinning sensation of surrounding, 3 episodes of vomiting, generalized body weakness, slurring of speech, drowsiness for six hours. Patient's symptoms progressive worsening over 5 hours with lethargic, severe bulbar symptoms of dysarthria, dysphagia, 


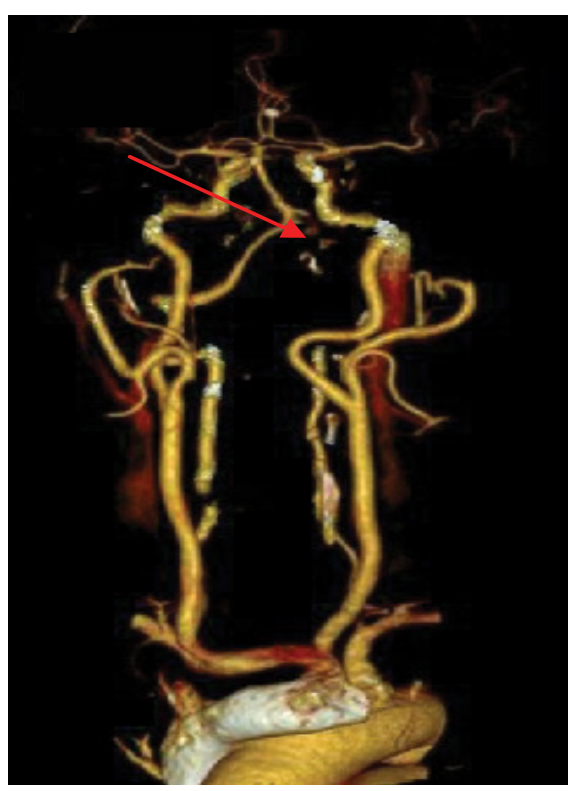

Figure 1: CT angiography showed left side distal vertebral artery (V3, V4 segment) severe stenosis and wall calcification. bilateral facial weakness, dyspnea, and quadriparesis. Neurological examination revealed lethargic, severe slurred dysarthric speech, cranial nerves examination showed $2 \mathrm{~mm}$ equal size pupils and reactive to light, bilateral facial muscles weakness (LMN), bilateral diminished gage reflex.

The strength of upper and lower extremity was $2 / 5$ (MRC grading) with increased tone and intact deep tendon reflexes while bilateral up going planter's response. Pinprick and proprioception sensation of limbs were not assessed due to poor conscious level of patient. Patient conscious level getting worsening to obtunded. Patient was intubated to protect airway as patient was poor to cough reflex and swallow assessment. Initial differential diagnosis of brainstem acute stroke, GBS, Myasthenia gravis crisis, botulism and rhombencephalitis. Past medical history of ischemic heart disease, newly diagnosed DM-II. Initial CT head, perfusion and angiography showed left side distal vertebral artery (V3, V4 segment) severe stenosis and
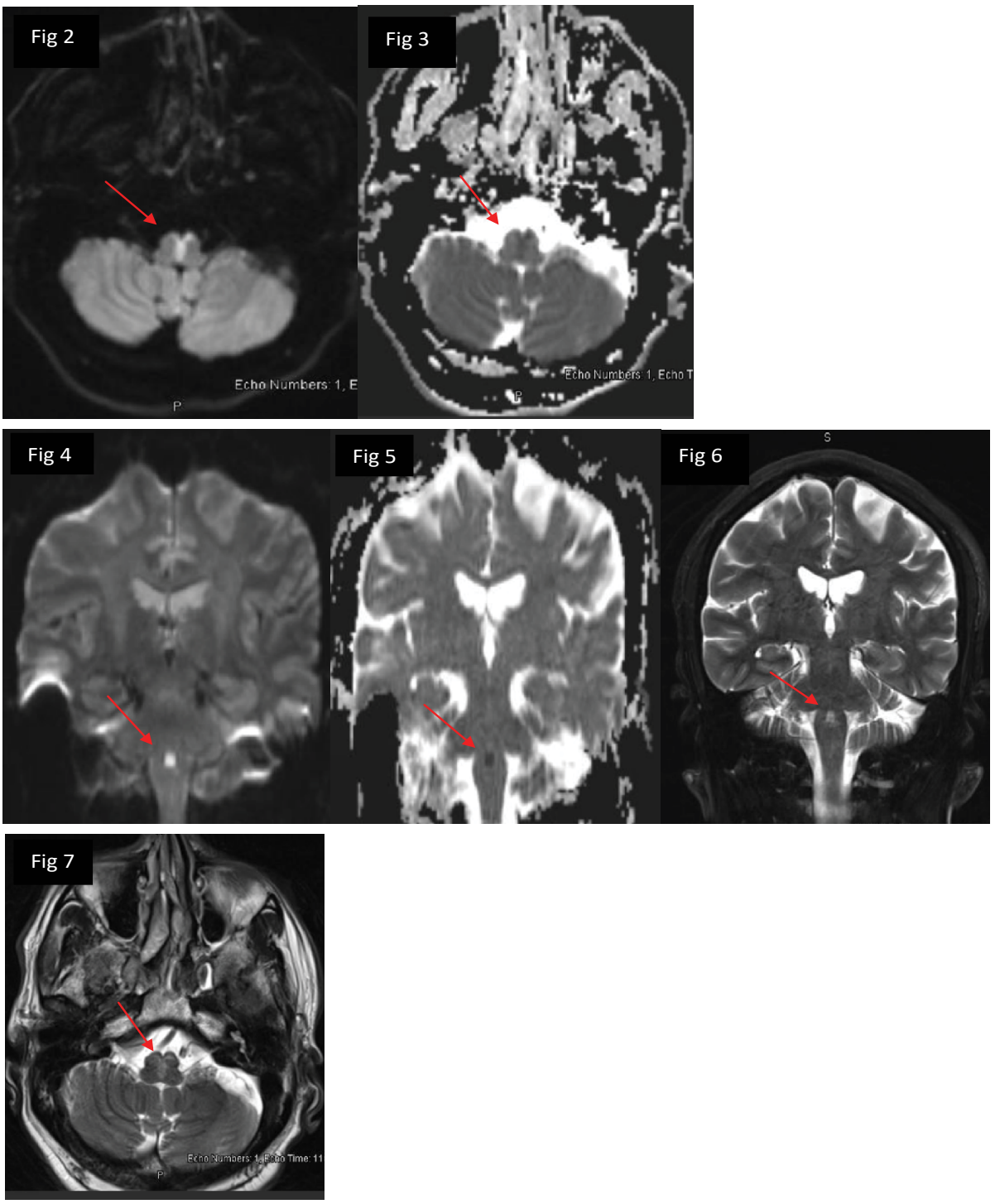

Figures 2-7: MRI head DWI/T2WI showed central bilateral medullary acute infarct extending to the bilateral pyramidal tracts ("heart shape sign"). 
wall calcification (Figure 1). MRI, MRA head showed central bilateral medullary acute infarct extending to the bilateral pyramidal tracts ("heart shape sign") as shown in (Figure 2, Figure 3, Figure 4, Figure 5, Figure 6 and Figure 7) in DWI, ADC, TWI, there is occlusion of left distal vertebral artery $\mathrm{V} 4$ segment most likely by thrombus as (Figure 8 and Figure 9). The patient was started Aspirin, atorvastatin, LMWH for DVT prophylaxis, antibiotic for aspiration pneumonia and oral hypoglycemia agents.

Patient CBC, CMP, PT, APPT, were normal. His random serum glucose on admission was $9.5, \mathrm{HbA1c}$ was $8.1 \%$, Cholesterol of $3.7 \mathrm{mmol} / \mathrm{L}$ with LDL level of $2.4 \mathrm{mmol} / \mathrm{L}$, Troponin T was $6.9 \mathrm{ng} / \mathrm{L}$. CSF analysis showed WBC of 01 , protein of $0.24 \mathrm{gm} / \mathrm{L}$, glucose of $6.7 \mathrm{mmol} / \mathrm{L}$, CSF AFB, culture showed no organism seen, CSF -TB PCR, CSF HSV1,2 PCR, VZV PCR were negative. Echo showed EF $54 \%$, no contrast passage after Valsalva maneuver, 48-hour holter study showed no evidence of atrial fibrillation. after three days, patient was extubated and transfer to stroke unit. After 5 days in stroke unit with stable course, patient shifted to rehabilitation center for muscle strengthening exercises, gait, speech, and swallow training and discharged to home after 10 days with modified rankin scale of $2 / 6$. The possible stroke mechanism in our patient is intracranial left distal vertebral artery occlusion. In contrast to unilateral medial medullary infarction, patients with bilateral medial medullary infraction have poor prognosis in view of complicated with quadriparesis and respiratory failure.

\section{Discussion}

$20 \%$ of ischemic strokes in the brain involve posterior circulation structures (vertebrobasilar circulation). The most common causes of posterior circulation large artery ischemia are atherosclerosis, embolism, and dissection. Less than $1 \%$ of all posterior circulation infarctions present as medial medullary syndrome. In medial medullary stroke, the most consistent finding is a contralateral hemiparesis. Ipsilateral tongue paralysis is the least common but most topographically localizing sign of medial medullary infarction. It results from the occlusion of one of the paramedian perforating of the anterior spinal artery or vertebral arteries. Clinical trial for medial medullary infarction including of ipsilateral hypoglossal paralysis, contralateral hemiparesis sparing the face, and contralateral loss of deep sensation. Bilateral medial medullary stroke is a very rare type of stroke. The most common symptoms are dysarthria, hypoglossal palsy, flaccid, or spastic quadriplegia. The characteristic brain MRI finding of "heart shape sign" at DWI noted. In a systematic review of 38 patients with bilateral medial medullary infraction, $23.8 \%$ of patients died and $61.9 \%$ survivors remained dependent. This patient presented with sudden onset of bulbar sign and symptoms, quadriparesis and respiratory failure. CSF study showed no evidence of infection or inflammation. MRI head revealed of "heart shape sign" in DWI suggestive of bilateral medial medullary stroke with left distal vertebral artery occlusion/ thrombus noted. Patient was intubated for protect airway and worsening conscious level of obtunded. He was extubated after 3 day and shift to stroke ward and rehabilitation center. After 10-day, patient discharge on modified rankin scale of $2 / 6$. This case study helps in early recognition of bilateral medial medullary stroke presented with clinical trial of sudden onset of bulbar sign and symptoms, quadriparesis and respiratory failure that mimics of GBS, Botulism, Myasthenia gravis crisis, brainstem encephalitis etc.

\section{Conclusion}

Bilateral medial medullary stroke is very rare type of posterior circulation stroke that presented with clinical trial of sudden onset of bulbar signs and symptoms, quadriparesis and respiratory failure. GBS and Neuromuscular disorders are important differential diagnosis of bilateral medial medullary stroke. Majority

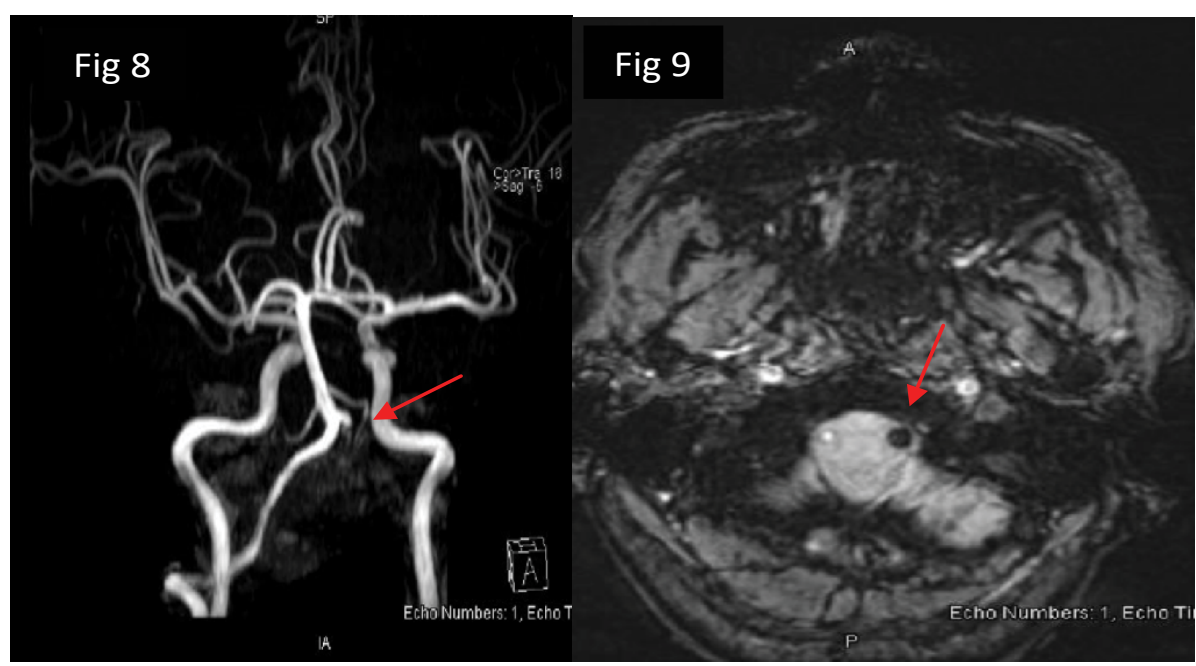

Figures 8-9: MRA (TOF) and SWI head showed occlusion of left distal vertebral artery V4 segment most likely by thrombus. 
of patient with bilateral medial medullary stroke who survivors remained dependent.

\section{Conflict of Interest}

The authors declare that they have no conflict of interest.

\section{Funding Disclosure}

No funding was received for this study.

\section{Author's Contribution}

Liaquat Ali: Data collection, data analysis, manuscript writing \& lectures review; Numan Amir: Data analysis, manuscript writing; Naveed Akhtar: Data analysis, manuscript writing \& lectures review; Osama Elalamy: Data analysis, manuscript writing, lectures review; Mohammed Alhatou: Manuscript writing \& lectures review; Ghulam Adeli: Manuscript writing, lectures review; Ambreen Iqrar: Manuscript writing, lectures review.

\section{Data Availability}

The authors declare that data supporting the findings of this study are available within the article.

\section{Protocol IRC ID- MRC -04-21-563}

Entitled "The Heart Shape-Sign in Bilateral Medial Medullary Infarction; Complicated with Quadriparesis and Respiratory Failure" has been approved by IRC HMC-Qatar.

\section{Ethical Approval}

All procedures performed in the studies involving human participants were in accordance with the ethical standards of the institutional and/or national research committee and with the 1964 Helsinki Declaration and its later amendments or comparable ethical standards.

\section{Informed Consent}

Informed consent was obtained from all individual participants included in the study.

\section{References}

1. Louis R Caplan, Robert J Wityk, Thomas A Glass, Jorge Tapia, Ladislav Pazdera, et al. (2004) New england medical center posterior circulation registry. Ann Neurol 56: 389398.
2. Caplan LR (2015) Vertebrobasilar Ischemia and Hemorrhage: Clinical Findings, Diagnosis and Management of Posterior Circulation Disease. ( $2^{\text {nd }}$ edn), Cambridge University Press, Cambridge.

3. D C Brewster, A C Moncure, R C Darling, J J Ambrosino, W M Abbott (1985) Innominate artery lesions: Problems encountered, and lessons learned. J Vasc Surg 2: 99-112.

4. R Labauge, M Boukobza, M Pagès, J M Blard, J Dimitrijevic, et al. (1987) [Occlusion of the vertebral artery (100 personal cases)]. Rev Neurol (Paris) 143: 490-509.

5. Caplan LR (2008) Dissections of brain-supplying arteries. Nat Clin Pract Neurol 4: 34-42.

6. Kim JS (2003) Pure lateral medullary infarction: Clinicalradiological correlation of 130 acute, consecutive patients. Brain 126: 1864-1872.

7. K-D Choi, H-Y Shin, J S Kim, S-H Kim, O-K Kwon, et al. (2005) Rotational vertebral artery syndrome: Oculographic analysis of nystagmus. Neurology 65: 1287-1290.

8. Pessin MS, Gorelick PB, Kwan ES, Caplan LR (1987) Basilar artery stenosis: Middle and distal segments. Neurology 37: 1742-1746.

9. Voetsch B, DeWitt LD, Pessin MS, Caplan LR (2004) Basilar artery occlusive disease in the New England medical center posterior circulation registry. Arch Neurol 61: 496-504.

10. Caplan LR (1980) "Top of the basilar" syndrome. Neurology 30: 72-79.

11. Caplan LR, Hedley-Whyte T (1974) Cuing and memory dysfunction in alexia without agraphia. A case reports. Brain 97: 251-262.

12. Fisher CM (1982) Disorientation for place. Arch Neurol 39: 33-36.

13. Tyler KL, Sandberg E, Baum KF (1994) Medical medullary syndrome and meningovascular syphilis: A case report in an HIV-infected man and a review of the literature. Neurology 44: 2231-2235.

14. Bassetti C, Bogousslavsky J, Mattle H, Bernasconi A (1997) Medial medullary stroke: Report of seven patients and review of the literature. Neurology 48: 882-890.

15. Dejerine J, Edward B Schlesinger (1914) Semiology of affections of the nervous system. Masson, Paris, 226-230.

16. Ishizawa K, Ninomiya M, Nakazato $Y$, Yamamoto T, Araki N (2012) "Heart appearance" infarction of the pons: A case report. Case Rep Radiol.

17. Pongmoragot J, Parthasarathy S, Selchen D, Saposnik G (2013) Bilateral medial medullary infarction: A systematic review. J Stroke Cerebrovasc Dis 22: 775-780. 